\title{
A case of spuriously high CK-MB: Contemplate beyond cardiac
}

\section{Rishard Abdul ${ }^{1}$, Michael Omar ${ }^{1}$, Shivananda Nayak², Surujpal Teelucksingh ${ }^{3}$, and Ronan Ali3}

1. Department of Internal Medicine, Medical Associates Hospital, St Joseph, Trinidad and Tobago

2. Department of Preclinical Sciences, University of the West Indies, St Augustine, Trinidad and Tobago

3. Adult Medicine Unit, Department of Clinical Medical Sciences, University of the West Indies, St Augustine,

Trinidad and Tobago

\section{CASE STUDY}

Please cite this paper as: Abdul R, Omar $M$, Nayak $S$, Teelucksingh S, Ali R. A case of spuriously high CK-MB: Contemplate beyond cardiac. AMJ 2017;10(10):890-893.

https://doi.org/10.21767/AMJ.2017.3122

Corresponding Author:

Michael Omar

Corner Albert and Abercromby Street

Medical Associates Hospital

St Joseph, Trinidad and Tobago

Email: mike.b.omar@gmail.com

\section{ABSTRACT}

Creatine kinase-MB (CK-MB) is a widely tested enzyme in cardiac disease and thus has important clinical implications.

We relate the scenario of a young patient presenting with chest pain whose CK-MB levels remained inordinately elevated despite a normal total CK level, resolution of symptoms and exclusion of coronary artery disease.

Further analysis by electrophoresis revealed the presence of a rare molecular variant of creatine kinase: macro-CK type 1. While this may be a benign finding, there are sparse data demonstrating an association with non-cardiac pathologies such as malignancy, endocrinopathies, connective tissue and autoimmune diseases.

Thus, after further exclusion of these associated conditions, the patient was reassured on the likely benign nature of macro-CK in this case.

\section{Key Words}

Cardiology, emergency medicine, biochemistry

\section{Implications for Practice:}

\section{What is known about this subject?}

There are rare and poorly understood macro-molecular variants of creatine kinase which cloud the encountering physicians' next steps in management.

\section{What new information is offered in this case study?}

The macro-molecular variant of creatine kinase may be incidentally discovered in a patient without coronary artery disease and should prompt screening for associated noncardiac conditions.

3. What are the implications for research, policy, or practice?

Patients with persistently abnormal creatine kinase assays, in whom coronary artery disease has been excluded, may be screened for macro-molecular variants of creatine kinase and their known associated conditions.

\section{Background}

Chest pain is a common presenting complaint to the emergency department and exclusion of acute coronary syndromes is imperative given its high prevalence. In such circumstances, despite the superior value of troponin estimation, cardiac biomarkers such as creatine kinase and its $\mathrm{MB}$ isoform remain widely requested.

In very rare instances, patients may present with persistent and/or inordinate elevations of CK-MB even though cardiac disease has been thoroughly ruled out. Moreover, the CK$\mathrm{MB}$ result may even paradoxically exceed the total CK.

We aim to shed light on this clinical dilemma by raising the awareness of a macro-molecular variant of creatine kinase (macro-CK) which may produce such a scenario.

Although the data is yet sparse on this esoteric molecule, its identification in the serum may further prompt relevant screening for purported non-cardiac disease associations. 


\section{Case details}

Our case involves a 27-year-old, previously well, East Indian woman who complained of central chest pain suggestive of gastro-oesophageal reflux disease (GERD) for a two-day period. Physical examination was unremarkable and electrocardiogram (ECG) showed no evidence of myocardial ischemia or injury. There was no evidence of conduction abnormalities including new left bundle branch block. The patient was treated with proton pump inhibition with good clinical response.

However, as part of the emergency department work up, cardiac biomarkers revealed the unusual and initially inexplicable situation in which CK-MB was markedly elevated 225U/L (reference range: 1-24U/L) exceeding that of the total CK which was only $128 \mathrm{U} / \mathrm{L}$ (reference range: 30-170U/L) and yielding a CK-MB: total-CK ratio of 1.75 . Although initial and six-hour interval troponin $T$ remained negative, out of an abundance of caution, myocardial ischemia was effectively ruled out on stress echocardiography.

The cardiac biomarkers were reassessed four and eight weeks later when the patient was asymptomatic. These exhibited a persistently high CK-MB: total-CK ratio of 200:101U/L at four weeks and, 202:104U/L at eight weeks respectively (see Table 1). Subsequent CK electrophoresis demonstrated the presence of a macromolecular variant of CK (Macro CK type 1).

Appropriate screening excluded endocrinopathies such as thyroid, adrenal and ovarian disorders by estimation of free $\mathrm{T}_{4}\left(\mathrm{FT}_{4}\right)$, thyroid stimulating hormone $(\mathrm{TSH})$, electrolyte levels, luteinizing hormone (LH) and follicle stimulating hormone (FSH) titres. Neoplastic diseases such as breast, cervical and colonic malignancy were ruled out by means of breast ultrasound, Pap smear and faecal occult blood testing. Autoimmune conditions were excluded by means of assessing erythrocyte sedimentation rate (ESR), C- reactive protein (CRP) and Anti-Nuclear Antibody (ANA) titres. Given the negative screening assessment, the patient was then reassured on the likely benign, incidental nature of macroCK in this case.

\section{Discussion}

Creatine kinase (CK) is a muscle enzyme consisting of two cytosolic subunits, $M$ and $B$. There are three (3) recognized isoenzymes: CK-MM (skeletal muscle), CK-BB (brain) and CKMB (heart), each being an 80kDa dimer. ${ }^{1}$ Certain higher than normal molecular weight forms of these enzymes have been described but are relatively rare. ${ }^{2,3}$ These are known as macro-enzymes and carry a molecular weight of greater than 200kDa., Macro molecular forms of not only creatine kinase have been described but also that of lactate dehydrogenase, amylase and aspartate aminotransferase. ${ }^{5}$ These molecules provide diagnostic challenges for physicians as they often lead to an interference in the biochemical assay for their respective enzymes. This may result in confounded false positive diagnoses such as myocardial ischemia in this case.

The macro-molecular forms of creatine kinase are termed macro-CK type 1 and macro-CK type 2. Macro-CK type 1 is a complex formed by one of the CK isoenzymes and immunoglobulin in an antigen - (auto) antibody reaction. ${ }^{6}$ Most often, its components entail CK-BB and monoclonal IgG. ${ }^{2,6}$ Thus, this amalgamated molecule may interfere with commonly used biochemical assays by adding to the estimation of CK-MB but not to the total CK. ${ }^{7}$ Conversely, high levels of total CK with a normal CK-MB have also been described in the literature. ${ }^{1,2}$

Despite a meagre estimated prevalence of 0.9-1.2 per cent, ${ }^{2}$ researchers have discovered an association with macro-CK type 1 and various disorders including hypothyroidism, neoplasia, autoimmune conditions, myositis and cardiovascular disease. ${ }^{7}$ Persuasively, Lee et al. showed that more than 50 per cent of his patients with macro CK type 1 had some form of myositis, whether it was autoimmune, malignancy associated or drug induced $(p<0.0005)$, which seems to be somewhat reproducible in other studies. ${ }^{2}$ As in our patient, macro CK type 1 may occur in apparently healthy individuals ${ }^{8}$ and interestingly, is more common in females. ${ }^{2,8}$ Macro-CK has additionally been seen in children ${ }^{3}$ in association with myocardial damage. Rödöö, ${ }^{9}$ further described a paediatric case series of creatine kinase abnormalities in children with benign paroxysmal vertigo however there were no investigations done for macro-CK.

Macro-CK type 2 on the other hand is a nonimmunoglobulin bound macro-enzyme of mitochondrial origin with an estimated prevalence of 0.5-3.7 per cent. ${ }^{10}$ Overwhelming data has strongly linked the occurrence of macro CK type 2 to occult malignancy, ${ }^{1-8,10-12}$ particularly colorectal carcinoma, ${ }^{1,11}$ severe liver damage including cirrhosis and paediatric cardiac pathology. ${ }^{3}$ Furthermore, its presence in these pathologies may be considered as a poor prognostic indicator. ${ }^{2}$

Irrespective of the macro-CK subtype, a retrospective study by Chang et al. concluded that approximately 40 per cent of his patients with a CK-MB: total CK ratio greater than 1 had 
an underlying malignancy. The most frequent was colorectal cancer (16.5 per cent), followed by lung cancer (15.9 per cent) and hepatocellular carcinoma (14.5 per cent). ${ }^{1}$

Clinically, when considering the presence of macro-CK for a patient, it may be prudent to first thoroughly investigate for myocardial ischemia due to its high prevalence and consequences if overlooked. This should include a detailed clinical history, physical examination, along with relevant investigations such as echocardiography and stress testing. In the face of high clinical suspicion, more specific investigations such as coronary angiography may be warranted. For instance, one must consider the rare but highly fatal anomalous aortic origin of the coronary artery (AAOCA) in the young as a cause of myocardial ischemia and sudden cardiac death. ${ }^{13}$

Once cardiac pathology is excluded, the presence of macro $\mathrm{CK}$ in the serum may be determined using special methods such as immune-inhibition, chromatography or electrophoresis. ${ }^{12}$ If confirmed, appropriate screening for the above mentioned disease associations of macro-CK should ensue before reassuring patients.

Up to the time of writing, available data concerning macro CK demonstrate rare associations with various pathologies. In 2015, Chang et al. concluded that the CK-MB to total CK ratio could be used as a primary screening tool for certain malignancies, including those stated prior. However, neither this nor its use in screening for autoimmune disease has been reinforced due to the lack of sufficient sensitivity and specificity data. Further research into this rare molecule is needed to determine whether the presence of macro CK is considered a risk factor for malignancy and autoimmune disease as there are currently no prospective cohorts available to determine its relative risk.

\section{Conclusion}

It is important as clinicians that we have a fair awareness of macro-molecular enzyme variants when interpreting abnormal biochemical tests.

In the setting of patients with persistently abnormal creatine kinase levels, in whom cardiac disease has been excluded, it is worthwhile to investigate for macromolecular enzymes. The presence of these molecules should then prompt investigations for known disease associations. Patients may then be reassured, as in our scenario, of the likely benign nature of macro CK.

\section{References}

1. Chang C, Liou C, Su M, et al. Creatine Kinase (CK)-MB-toTotal-CK Ratio: A Laboratory Indicator for Primary Cancer Screening. Asian Pac J Cancer Prev. 2015;16(15):6599-6603.

2. Lee KN, Csako G, Bernhardt P, et al. Relevance of macro creatine kinase type 1 and type 2 isoenzymes to laboratory and clinical data. Clin Chem. 1994;40:12781283.

3. Wu AHB, Herson VC, Bowers GN Jr. Macro creatine kinase 1 and 2: Clinical significance in neonates and children as compared with adults. Clin Chem. 1983;29:201-204.

4. Liu C, Lai $Y, W u ~ Y$, et al. Macro-enzyme Creatine Kinase in the Era of Modern Laboratory Medicine. J Chin Med Assoc. 2010;73(1):35-39.

5. Galasso P, Litin S, O'Brien J. The Macroenzymes: A Clinical Review. Mayo Clin Proc. 1993;68(4):349-354.

6. Stein W, Bohner J, Krais J, et al. Macro creatine kinase $B B$; evidence for specific binding between creatine kinase BB and immunoglobulin G. J Clin Chem. 1981;19:925-930.

7. Maghamiour N, Safaie N. High creatine kinase (CK)-MB and lactate dehydrogenase in the absence of myocardial injury or infarction: A case report. J Cardiovasc Thorac Res. 2014;6(1):69-70.

8. Whelan PV, Malkus $\mathrm{H}$. A macro $\mathrm{CK}$ isoenzyme in serum of apparently healthy individuals. Clin Chem. 1983;29:1411-14.

9. Rödöö $P$, Hellberg D. Creatine kinase $M B(C K-M B)$ in benign paroxysmal vertigo of childhood: $A$ new diagnostic marker. J Pediatr. 2005;146(4):548-551.

10. Stein W, Bohner J, Renn W, et al. Macro creatine kinase type 2: Results of a prospective study in hospitalized patients. Clin Chem. 1985;31:1959-1964.

11. Mercer DW, Talamo TS. Multiple markers of malignancy sera of patients with colorectal carcinoma: preliminary clinical studies. Clin Chem. 1985;31:1824-1828.

12. Laureys $\mathrm{M}$, Sion JP, Slabbynck $\mathrm{H}$, et al. Macromolecular creatine kinase type 1: A serum marker associated with disease. Clin Chem. 1991;37:430-34.

13. Peñalver J, Mosca R, Weitz D, et al. Anomalous aortic origin of coronary arteries from the opposite sinus: $A$ critical appraisal of risk. BMC Cardiovasc Disord. 2012;12(1).

\section{PEER REVIEW}

Not commissioned. Externally peer reviewed. 


\section{CONFLICTS OF INTEREST}

The authors declare that they have no competing interests.

\section{FUNDING}

None

\section{PATIENT CONSENT}

The authors, Abdul R, Omar M, Ali R, Nayak S, Teelucksingh $S$, declare that:

1. They have obtained written, informed consent for the publication of the details relating to the patient(s) in this report.

2. All possible steps have been taken to safeguard the identity of the patient(s).

3. This submission is compliant with the requirements of local research ethics committees.

Table 1: Cardiac biomarkers at 4-week and 8-week interval respectively

\begin{tabular}{|l|l|l|l|}
\hline Time Period & $\begin{array}{l}\text { CK } \\
\text { (NR 30- } \\
\mathbf{1 7 0} \text { U/L) }\end{array}$ & $\begin{array}{l}\text { CK-MB } \\
\text { (NR 1- } \\
\text { 24U/L) }\end{array}$ & $\begin{array}{l}\text { CK-MB: } \\
\text { total-CK } \\
\text { ratio }\end{array}$ \\
\hline $\begin{array}{l}\text { Initial } \\
\text { presentation }\end{array}$ & 128 & 225 & 1.75 \\
\hline $\begin{array}{l}\text { Week 4 follow } \\
\text { up }\end{array}$ & 101 & 200 & 1.98 \\
\hline $\begin{array}{l}\text { Week 8 follow } \\
\text { up }\end{array}$ & 104 & 202 & 1.94 \\
\hline
\end{tabular}

